\title{
BASES PARA LA GOBERNANZA DEL DISTRITO DE CIENCIA, TECNOLOGÍA E INNOVACION EN MEDELLÍN, COLOMBIA*
}

\author{
Recibido: 15 de julio de 2015 • Aprobado: 10 de noviembre de 2015 \\ Carlos Mario Molina Betancur* \\ Jorge Andrés Polanco López De Mesa*** \\ Juan Manuel Montes Hincapié ${ }^{* * *}$
}

\section{RESUMEN}

El objetivo de este trabajo es analizar las bases de gobernanza del Distrito de ciencia, tecnología e innovación de Medellín en Colombia, mediante una revisión de literatura e integración de trabajos inéditos de los autores para ilustrar aspectos claves de la administración de este nuevo arreglo institucional. Los resultados muestran que la gobernanza del Distrito se basa en la lectura de las dinámicas y tendencias territoriales, la integración de intereses entre los agentes y la gestión de la ciencia, la tecnología y la innovación.

\section{PALABRAS CLAVE}

Gestión de la tecnología y la innovación; Innovación social; Planificación y políticas de desarrollo regional

\section{CLASIFICACIÓN JEL}

O32, 035, R58

\section{CONTENIDO}

Introducción; 1. La gobernanza y el distrito de Ciencia, Tecnología e Innovación; 2. Bases para la gobernanza del nuevo distrito de Ciencia, Tecnología e Innovación; 3. Conclusiones; Bibliografía.

\footnotetext{
Este trabajo es un artículo de revisión que busca integrar resultados inéditos de investigaciones realizadas por los autores: 1) Medellín, ciudad más innovadora: una visión desde la academia; 2) El bicentenario constitucional colombiano; 3) Consultoría para el Área Metropolitana del Valle de Aburrá; 4) Tesis doctoral L'État à l'épreuve de la gouvernance.

** Abogado, Universidad de Medellín, Medellín, Colombia. Especialista en derecho administrativo y derecho procesal, Universidad París II, París, Francia. Magister en derecho público interno, Universidad París II, París, Francia. Doctor en Derecho Público, Universidad París II, París, Francia. Profesor titular, Facultad de Derecho, Universidad de Medellín. Miembro del Grupo de Investigaciones Jurídicas, Universidad de Medellín, Carrera 87 No. 30-65, oficina 12-106, Teléfono +574. 3405482, Medellín, Colombia. Correo electrónico: cmolina@ udem.edu.co.

** Ingeniero civil, Universidad Nacional de Colombia, Medellín, Colombia. Magister en Environnement, Temps, Spaces, Societes, Université d'Orléans, Francia. Doctor en Socio-economía del desarrollo, Escuela de Altos Estudios en Ciencias Sociales de Paris (EHESS), París, Francia. Profesor asistente, Facultad de Ciencias Económicas y Administrativas, Universidad de Medellín. Miembro del Grupo de Investigación en Cultura y Gestión Organizacional (CyGO) y del Grupo de Investigación en Sostenibilidad (GIS), Universidad de Medellín, Carrera 87 No. 30-65, oficina 12-104, Teléfono +574.3405555 (ext. 5602) Medellín, Colombia. Correo electrónico: japolanco@udem.edu.co.

*.** Ingeniero químico, Universidad de Antioquia, Medellín, Colombia. Especialista en alta gerencia con énfasis en calidad, Universidad de Antioquia, Medellín, Colombia. Magister en Gestión Tecnológica, Universidad Pontificia Bolivariana, Medellín, Colombia. Doctor en Proyectos de Innovación Tecnológica en la Ingeniería de Producto y Proceso, Universitat Politécnica de Catalunya (UPC), Barcelona, España. Profesor asistente, Facultad de Ciencias Económicas y Administrativas, Universidad de Medellín. Líder del Grupo de Investigación en Ciencia, Tecnología, Innovación y Emprendimiento (CITIE), Universidad de Medellín, , Cra. 87 No. 30-65, oficina 12-101, Teléfono +574.3405450, Medellín, Colombia. Correo electrónico: jmontes@udem.edu.co.
} 


\section{GOVERNANCE BASIS FOR THE SCIENCE, TECHNOLOGY AND INNOVATION DISTRICT IN MEDELLIN, COLOMBIA}

\section{ABSTRACT}

The goal of this paper is to analyze governance basis of the Science, Technology and Innovation district in Medellin, Colombia through a literature review and the integration of unpublished works done by the authors in order to illustrate key aspects of the administration of this new institutional arrangement. Results show that the District's governance is based on the understanding of territorial dynamics and trends, the integration of interests between the management agents and Science, Technology and Innovation management.

\section{KEY WORDS}

Technology and innovation management; Social innovation; Planning and regional development policies

\section{JEL CLASSIFICATION}

O32, 035, R58

\section{CONTENT}

Introduction; 1. Governance and the Science, Technology and Innovation; 2. Basis for the Governance of the new Science, Technology and Innovation district; 3. Conclusions; Bibliography.

\section{BASES PARA A GOVERNANÇA DO DISTRITO DE CIÊNCIA, TECNOLOGIA E INOVAÇÃO EM MEDELLÍN, COLÔMBIA}

\section{RESUMO}

O objetivo deste trabalho é analisar as bases de governança do Distrito de ciência, tecnologia e inovação de Medellín na Colômbia, mediante uma revisão de literatura e integração de trabalhos inéditos dos autores para ilustrar aspectos chaves da administração deste novo acordo institucional. Os resultados mostram que a governança do Distrito se baseia na leitura das dinâmicas e tendências territoriais, a integração de interesses entre os agentes e a gestão da ciência, a tecnologia e a inovação.

\section{PALAVRAS CHAVE}

Gestão da tecnologia e a inovação; Inovação social; Planificação e políticas de desenvolvimento regional

\section{CLASSIFICAÇÃO JEL}

O32, 035, R58

\section{CONTEÚDO}

Introdução; 1. A governança o distrito de Ciência, Tecnologia e Inovação; 2. Bases para a governança do novo distrito de Ciência, Tecnologia e Inovação; 3. Conclusões; Bibliografia. 


\section{INTRODUCCIÓN}

Medellín fue reconocida en 2013 como la ciudad más innovadora del mundo, en el concurso city of the year, organizado por el Wall Street Journal (2013) gracias al fomento de políticas de inclusión social, un moderno sistema de transporte público y políticas ambientales innovadoras.

El progreso y la inclusión social han sido impulsados con el apoyo del Grupo Empresas Públicas de Medellín (EPM) a la Educación, a la creación de espacios culturales (parque explora, jardín botánico, parques biblioteca, Centro Cultural de Moravia), a la gestión de servicios públicos, al desarrollo social de zonas marginadas, a la reducción de la criminalidad, a la propuesta de museos y centros culturales, bibliotecas y escuelas públicas.

Por su parte, el moderno sistema de transporte público se basa en un Sistema Integrado de Transporte (SIT) - metro, buses y metrocables - y la política ambiental ha logrado disminuir las emisiones de $\mathrm{CO}_{2}$.

Bajo este contexto y con el liderazgo de la Corporación Ruta N, creada para promover negocios innovadores basados en tecnología, ahora la ciudad de Medellín asume el nuevo reto de convertirse en la ciudad más innovadora de Latinoamérica en el 2021, donde la ciencia, la tecnología y la innovación se convierten en motores fundamentales para su desarrollo. Como lo plantea esta Corporación (2014d), es con este propósito que la ciudad quiere crear el nuevo distrito metropolitano de ciencia, tecnología e innovación.

En Colombia existen cinco distritos que se distinguen por las diferentes vocaciones del territorio y a los cuales vendría a sumarse el distrito mencionado: Distrito capital; Distrito Industrial y portuario de Barranquilla; Distrito turístico y cultural de Cartagena; Distrito portuario, Biodiverso, Industrial y Ecoturístico de Buenaventura; y Distrito Turístico, Cultural e Histórico de Santa Marta.

Es decir, que de acuerdo con Suelt-Cock y Osorio (2011), se está en presencia del surgimiento de un nuevo orden territorial, el cual necesita, según Prats (2005) y Le Galès (1995), grandes acuerdos políticos y amplios parámetros de gobernanza. El distrito emergente, busca reagrupar la administración de los servicios del área metropolitana del valle de Aburrá, teniendo en cuenta la participación de actores públicos y privados en la formulación y ejecución de políticas públicas y partiendo de la autonomía local derivada de la descentralización político-administrativa del país, según lo plantea Molina-Betancur (2011).

El objetivo de este trabajo es analizar las bases de gobernanza del nuevo distrito de ciencia, tecnología e innovación, mediante la integración de resultados inéditos 
de los autores para ilustrar aspectos claves de la administración de este nuevo arreglo institucional.

Así pues, en este texto se presenta en primer lugar la relación teórica entre gobernanza y territorio, con base en ciencia, tecnología e innovación. Luego se esbozan los elementos metodológicos para, finalmente, analizar tres aspectos básicos de gobernanza: la lectura de las dinámicas y tendencias territoriales, la integración de intereses entre los agentes del distrito y la gestión de la ciencia, la tecnología y la innovación. Finalmente, se presentan las conclusiones del trabajo resaltando los principales hallazgos y proponiendo futuras investigaciones.

\section{LA GOBERNANZA Y EL DISTRITO DE CIENCIA, TECNOLOGÍA E INNOVACIÓN}

En Colombia, el área metropolitana es una figura constitucional nueva, que pertenece al campo del ordenamiento territorial, el cual se concibe como una política de Estado para garantizar un proceso planificado del territorio desde los ámbitos económico y social, con implicaciones políticas, técnicas y administrativas.

Según Massiris-Cabeza (1993), el objeto regional de esta nueva tendencia departamental, es la de organizar, armonizar y administrar la óptima ocupación y utilización del espacio público, de modo que estos procesos modernos contribuyan adecuadamente al desarrollo humano armónico y sostenible.

Esta planeación integral, hace parte de un concepto de desarrollo institucional endógeno regional que, como lo expresan Agudelo, Medina y Álvarez-García (2010), fortalece la asociación entre diferentes actores de la región como evidencias de avances en la transformación institucional del Área Metropolitana de Valle de Aburrá. De esta transformación surge una nueva relación entre la ciudad y su entorno regional, así como un nuevo modo de gobierno más horizontal como condición de sostenibilidad. Es por eso que el análisis de la transformación institucional se aborda desde el concepto de gobernanza territorial.

\subsection{La gobernanza territorial}

Desde hace unas dos décadas, se habla sobre la sostenibilidad y su relación con la gobernanza territorial: a través de la gobernanza se propendería por establecer de manera confiable un desarrollo sostenible. El informe Brundtland (1987) globalizó este nuevo concepto de desarrollo, denotándolo como un proceso orientado al bienestar de la población actual sin comprometer el bienestar de las generaciones futuras. Sin embargo, según Polanco (2009), es claro que el camino por recorrer en su práctica local apenas comienza. 
Esta práctica local es un proceso de aprendizaje que debe ser monitoreado y mejorado, como lo sugieren la OECD (2011 y 2001) y Stagl (2007). En ella participan actores gubernamentales, empresariales y sociales, cuyas relaciones pueden ser informales o formales. De acuerdo a North (1991), dichas relaciones pueden considerase reglas de juego o instituciones. Desde esta perspectiva, autores como Dovers y Hezri (2010), Soderbaum (2009) y Fischer, Petersen y Feldkoetter (2007), consideran que las instituciones son fundamentales en la práctica del desarrollo sostenible.

Así las cosas, las escalas regional y metropolitana, pueden conjugarse alrededor del concepto de Ciudad-Región. Más allá de la visión centro-periferia asociada al desarrollo desigual que plantea Rodríguez (2001), la Ciudad-Región se configura por relaciones de interdependencia entre el área metropolitana y su entorno regional y por economías de aglomeración que tienden a generar retornos crecientes y ventajas competitivas para los productores locales, según Scott (2001).

Ahora bien, esta configuración es sensible a la expansión urbana, pues tiende a aumentarse la brecha entre ricos y pobres (fragmentación espacial), la demanda de servicios públicos e infraestructura vial y, además, a ejercerse mayor presión sobre los ecosistemas. Lo cual necesita solucionarse, según Wheeler (2009), teniendo en cuenta un soporte organizacional que vincule varios niveles del Estado y múltiples sectores de la sociedad.

En los casos latinoamericanos (Chile, México y Colombia) documentados por Orellana $(2009,2012)$ y por Yory (2011), estos problemas de sostenibilidad se evidencian sobre todo en la segregación espacial, la calidad ambiental y la fractura institucional. Los fenómenos de segregación espacial y de calidad ambiental adquieren todavía más importancia con respecto al impacto en el bienestar de la población, cuando el soporte institucional se encuentra fracturado, según Wheeler (2009). Y esta fractura será aún más evidente, en palabras de Yory (2011, p. 55), cuando "se restrinja el desarrollo regional a las posibilidades de ejecución presupuestal que éste brinda dentro de su fuero estrictamente jurisdiccional impidiendo, de tal forma que, por ejemplo, un departamento o municipio invierta en otro, aún a sabiendas de los beneficios que tal situación pudiera proporcionar a ambas instancias".

El problema regional así expresado es, sobre todo, institucional, y de naturaleza política, y proviene de la posición de las regiones en la jerarquía del Estado, de la fragmentación de las jurisdicciones y de las poblaciones en dichas regiones y de la resistencia política a diferentes formas de planificación.

De la misma manera lo plantea Orellana (2009) cuando argumenta que los gobiernos locales resisten a las agencias metropolitanas, especialmente en los 
países en donde el uso del suelo es tradicionalmente controlado localmente, como en Estados Unidos y Chile. Esto conlleva a la fragmentación de la metrópolis, dada la competencia entre jurisdicciones locales por el acceso a los impuestos y dada la dificultad de regular la calidad ambiental, los usos del suelo y la equidad social.

La sostenibilidad de la región, del área metropolitana o, más bien, de la CiudadRegión, puede así plantearse en términos de gobernanza. Como lo expresan Launay (2006) y Williamson $(1996,2000)$, la gobernanza se refiere entonces a una forma de gobierno en donde la dirección vertical y jerárquica da paso a relaciones más horizontales entre actores gubernamentales, productivos y sociales.

Desde la perspectiva urbana que defiende Le Galès (1995), el concepto de gobernanza surge por dos razones. Primero, el concepto de gobierno local cae en desuso como consecuencia de la interdependencia entre diferentes niveles del Estado, la transformación de escalas espaciales y la multiplicación de redes que atraviesan una autoridad local. Segundo, el Estado se ha vuelto un actor como los demás dentro del proceso de elaboración y ejecución de políticas públicas, a raíz de la diferenciación y autonomía de los subsistemas sociales. Por lo tanto, la problemática de la gobernanza se refiere a las interacciones entre el Estado y la sociedad y a los modos de coordinación y negociación que posibiliten la acción pública.

Con la adopción del concepto de gobernanza urbana, se corre el riesgo de borrar los conflictos y las ideologías y de pensar solo en la efectividad de las políticas públicas, como consecuencia de la coordinación y la negociación entre actores. Sin embargo; precisa Le Galès (1995), la evidencia de fragmentación del gobierno de las ciudades, indica que la autoridad local no aporta necesariamente coherencia y racionalidad. Mientras que esta fragmentación e irracionalidad pueden perdurar, otros elementos de coherencia pueden ser aportados por otros actores de la gobernanza.

\subsection{El distrito de ciencia, tecnología e innovación}

Como lo plantean Massiris-Cabeza (2011) y Patiño-Millán (2004), el área metropolitana del Valle de Aburrá (AMVA) es una comunidad dinámica que se transforma constantemente en el desarrollo del devenir regional, es un geoespacio tecnoregional que conoce unos linderos territoriales imaginados de carácter local que impacta económica y socialmente todo un país. En efecto, es en este territorio que la ciencia, la tecnología y la innovación son factores estratégicos de desarrollo y, por tanto, se propone la creación de un distrito como plataforma para el aprovechamiento de recursos y el fomento de capacidades y competencias.

De acuerdo con el testimonio de Josep Miquel Piqué (Funcionario del Ayuntamiento de Barcelona), publicado por Ruta N (2014a), en la actualidad la Alcaldía de 
Medellín a través de Ruta N avanza en la consolidación de un distrito en ciencia, tecnología e innovación para Medellín. Para lograr esta finalidad se ha tomado como base la experiencia lograda a través del Programa ERICA (España y sus regiones intercambian conocimiento con Antioquia) y se ha planteado un plan de transferencia del modelo22@de Barcelona al distrito de ciencia, tecnología e innovación en Medellín. En este sentido se ha desarrollado un plan de trabajo que tiene por objeto desarrollar un modelo específico para Ruta N a partir del modelo 22@ (modelo de referencia mundial), en el que se dan las claves de como las ciudades tienen que articularse para crear economías de conocimiento.

Ruta N, por su parte, tiene el encargo del desarrollo del distrito en ciencia, tecnología e innovación de la ciudad de Medellín con un plan que viene analizando la realidad preexistente en la que se encuentra la capa urbanística, la capa de innovación, la capa empresarial y la capa de talento; y ver el conjunto de agentes que ya están interviniendo. Por tanto, la actividad siguiente es comenzar a construir este modelo de Medellín como una nueva economía del conocimiento que permita asegurar que las capas urbanísticas y las capas económicas están dando soporte a la creación de empresas sobre la base de ciencia, tecnología e innovación.

Expone igualmente Josep Miquel Piqué, que en la actualidad existen organizaciones muy bien planteadas: responsables de planificación; empresas públicas que desarrollan infraestructuras muy bien ejecutadas; y universidades que están desarrollando programas de innovación y transferencia del conocimiento.

En síntesis, el conjunto de agentes es extremadamente oportuno y clave. El papel crítico es la interconexión de las capas, que es precisamente el reto que tiene Ruta N: asegurar la relación entre economía y territorio. Es el caso, por ejemplo, de los clústeres de Energía, Salud y Tecnologías de la Información y la Comunicación (TIC). Lo interesante es ver cómo estos clústeres están territorializándose, es decir, conectándose con las capacidades y competencias de las nuevas generaciones de profesionales que tendrán que incorporarse al distrito en ciencia, tecnología e innovación del AMVA.

De acuerdo con el testimonio del funcionario del Ayuntamiento de Barcelona, la finalidad es desarrollar un modelo con el cual Ruta N pueda facilitar la presencia de empresas tractoras, incubadoras de empresas de base tecnológica y centros de desarrollo tecnológico, que faciliten la creación de nuevas ecologías de innovación.

No se trata de replicar de forma igual el modelo, dice Josep Miquel Piqué, sino de entender las claves de cada una de las cadenas de valor involucradas: urbanística, de innovación, empresarial, de talento. Se trata entonces de, a partir del modelo 
22@, inferir un metamodelo que permita el desarrollo del modelo específico para la ciudad de Medellín y el AMVA de forma que esté conectado con su realidad y, por lo tanto, asegurar su liderazgo en la economía y la sociedad del conocimiento.

Finalmente, el verdadero reto es entender que son las cuatro capas mencionadas, que tienen que dialogar a través de mecanismos de gobernanza de toda la ecología de la innovación en el territorio.

\subsection{Metodología de trabajo}

Este trabajo se plasma en un artículo de revisión porque analiza, sistematiza e integra los resultados inéditos de los siguientes cuatro trabajos de los autores:

1. Memorias de la presentación realizada en el panel "Medellín ciudad más innovadora: una visión desde la academia", desarrollado en julio de 2013 en la Universidad de Medellín, con la participación de Ruta N (una visión desde el gobierno) y el Grupo Nutresa (una visión desde la empresa).

2. Investigación concluida en 2010, titula "El bicentenario Constitucional colombiano 1820-2010".

3. Memorias de consultoría para el Área Metropolitana del Valle de Aburrá (AMVA) en 2008 y 2009.

4. La tesis doctoral titulada "L'État à l'épreuve de la gouvernance", defendida el 22 de octubre de 2007 en la Ecole des Hautes Etudes en Sciences Sociales de Paris.

Las memorias de la participación en el panel permiten hacer un análisis del modelo y sistema de gestión del nuevo distrito frente a las condiciones exigidas por la Norma internacional UNE 166002, publicada por la Asociación Española de Normalización y Certificación -AENOR (2006). Toda la información utilizada para este propósito ha sido obtenida a través de la base de datos de la plataforma Scienti de Colciencias (2013), la página web del Comité Universidad Empresa Estado (CUEE) de Antioquia (2013) y la página web de Ruta N (2014b y 2014c).

Por su parte, la investigación sobre el bicentenario constitucional proporciona las bases jurídicas del objeto de estudio. Las memorias de la consultoría al AMVA permiten actualizar la información sobre las actividades diseñadas para manejar los flujos económicos en la Ciudad-Región y sobre las sinergias entre actores que resultan de la lógica política inherente a la consolidación del valle de Aburrá como Distrito. Finalmente, la tesis doctoral aporta el marco teórico sobre la gobernanza. 
Bases para la gobernanza del distrito de ciencia, tecnología e innovacion en Medellín, Colombia

\section{BASES PARA LA GOBERNANZA DEL NUEVO DISTRITO DE CIENCIA, TECNOLOGÍA E INNOVACIÓN}

La gobernanza del distrito se basa en la identificación de las dinámicas y tendencias socioeconómicas del territorio, la integración de intereses y la gestión de la ciencia, la tecnología y la innovación.

\subsection{Dinámicas y tendencias del territorio}

Para conseguir una reducción de la pobreza e inequidad entre los ciudadanos, y poder satisfacer sus necesidades básicas, todas las regiones deben apuntar a ser competitivas y a obtener un crecimiento económico sostenible. Para ello, dentro de los planes de desarrollo nacional, departamental y municipal en Colombia, se han definido la innovación, la ciencia, la tecnología y políticas de competitividad y productividad como principales medios para lograr dichos objetivos, así lo expresan Molina-Betancur y Polanco (2015).

La ciencia, la tecnología y la innovación se definen como medios para el desarrollo del país, de acuerdo a principalmente tres evidencias. En primer lugar, frente a la urgencia de modernizar y reformar el sistema de educación superior del país, las autoridades colombianas solicitaron hace tres años a la Organización para la Cooperación y el Desarrollo Económico (OCDE) y al Banco mundial establecer las fortalezas y las debilidades del sistema. El informe presentado por estas organizaciones (2012), resalta la importancia de fortalecer la calidad y pertinencia de los programas, de elaborar una estrategia sostenible de financiamiento de la expansión de la cobertura con equidad y de formar vínculos más estrechos entre las instituciones de educación superior y el mundo productivo.

En segundo lugar, el gobierno nacional, con base en dichas recomendaciones, y por ser aspirante a tercer país en Latinoamérica, como miembro de la OCDE, elaboró un proyecto de reforma a la ley 30 de 1993 sobre la Educación Superior, presentada al Congreso en 2011, como lo expresa el Ministerio de Educación Nacional (2011), la cual no tuvo éxito, pero sigue en discusión.

En tercer lugar, expertos mundiales como Salmi (2013) han recomendado al Ministerio de Educación Nacional, buscar soluciones innovadoras para el desarrollo del sistema colombiano de educación superior y establecer el emprendimiento de una reforma de fondo, concebida y aceptada como política del Estado, a largo plazo, y no como una reforma de un gobierno en particular limitada por el horizonte electoral clásico de corto plazo. 
En este contexto, el primer paso para generar impactos económicos y sociales de grandes proporciones, es consolidar una cultura de innovación dentro de las instituciones de educación técnica, tecnológica y superior y de las empresas en general. Para ello, la cultura de investigación e innovación debe iniciarse desde la educación primaria hasta la educación superior y debe existir una unión entre el sector empresarial, las instituciones de educación y la sociedad civil para que exista un correcto intercambio de información.

Si bien es cierto que en los últimos 20 años se constata un avance en ese sentido, también es cierto que actualmente en Colombia se puede observar un retardo en cuanto al desarrollo de la innovación, ciencia y tecnología comparándolo con otros países similares. Según el DNP (2010), mientras que Colombia destina el 0.2\% de su PIB a la ciencia, la tecnología y la innovación, otros países de la región destinan 0.5\% (Argentina), $0.7 \%$ (Chile) y $0.8 \%$ (Brasil).

Ahora bien, el modelo de desarrollo nacional presentado por el DNP (2007) está orientado a incrementar la productividad económica, con sentido humano y con equidad social, dentro del contexto de la globalización de la economía, del conocimiento y de las nuevas tecnologías de la información que responda a las exigencias de un desarrollo más humano, en condiciones de competitividad.

En el ámbito local, el Plan de Desarrollo del departamento de Antioquia (2012-2015), titulado "Antioquia la más educada", publicado por la Gobernación de Antioquia (2012) es la vía por medio de la cual transitará el nuevo vehículo de la prosperidad en este departamento, cuyo centro de actividades políticas y socioeconómicas está en el valle de Aburrá.

En efecto, el fomento de la educación a través de redes es la estrategia para integrar el desarrollo económico y social de la región. Es el caso de la red de ciencia y tecnología de Antioquia que espera la consolidación del Sistema general de Ciencia y Tecnología en donde la alianza Universidad-Empresa-Estado juega un papel muy importante para la planeación integral del Departamento.

Esto construye progresivamente un modelo integral de desarrollo de Antioquia que la consolida en su proyecto de región. Actualmente, existen programas municipales pilotos, como es el caso de Enlaza Mundos de la Alcaldía de Medellín, apoyado por la representación política de la región, según el Consejo de Medellín (2008), en el cual se busca una ciudad más competitiva en un contexto de internacionalización, incentivando la investigación y la innovación. A través de dicho programa se apoya la movilidad estudiantil para continuar estudios de postgrados y/o realizar pasantías en el exterior centrándose en egresados de pregrado de las Instituciones 
de Educación Superior (IES) ubicadas en los alrededores del área metropolitana del Valle de Aburrá.

Estos procesos de movilidad académica, que serán abiertos a todos los municipios del Valle de Aburrá, se efectúan con el fin de convertir dicha área en el polo de atracción de recursos humanos, tecnológicos e intercambio de estudiantes e investigadores extranjeros, permitiendo así que en todas las universidades exista una cultura de internacionalización. En este sentido una correcta formación en el idioma inglés, como lo expresa Universia (2011), juega un papel importante para el cumplimiento de estos objetivos; de igual forma, le permite a los estudiantes tener la oportunidad que al momento de finalizar sus estudios puedan aprovechar la oferta estudiantil.

Lo anterior se justifica por las siguientes ventajas planteadas por Agudelo, Medina y Álvarez-García (2010), que generan en el territorio la flexibilidad y la capacidad de transformación, tanto de sus instituciones como de sus agentes:

- Reducción de los costos de transacción y producción;

- Fortalecimiento de la confianza y cooperación entre los actores económicos (empresas, entidades públicas, sociedad civil);

- Favorecimiento de la formación de redes empresariales, locales como agrupaciones o clústeres, distritos industriales y alianzas estratégicas;

- Rentabilidad mayor de las empresas;

- Estimulación de la capacidad empresarial y los mecanismos de aprendizaje;

- Creación y estimulo de nuevos productos;

- Favorecimiento de la capacidad de los gobiernos locales para lograr objetivos comunes de política comunes;

- Facilitación los procesos de cambio en las formas como se gobiernan o rigen las relaciones de los actores locales;

- Estimulación al desarrollo urbano, la difusión de la innovación y el conocimiento;

- Facilitación de la creación y expansión de empresas y la formación de redes empresariales.

Con lo anterior, a futuro, el nuevo distrito necesitará de una mayor integración y coordinación de la asociación Universidad-Empresa-Estado, así como de los planes 
estratégicos que desarrolla, en asocio, con las entidades públicas municipales, regionales, nacionales e internacionales.

\subsection{La relación entre economía y territorio: una cuestión política}

Como lo plantean Molina-Betancur y Polanco (2015), desde la perspectiva del new city-regionalism de Harrison (2010), la relación entre economía y territorio que busca el nuevo distrito sería posible si se articulan las lógicas económica y política de la Ciudad-Región. La construcción política de la Ciudad-Región es un fenómeno más bien desconocido. Como lo plantea este último autor, aunque la Ciudad-Región es sensible a los flujos económicos y a las relaciones en red, también está determinada por el territorio definido en la malla político-administrativa. Es decir que, mientras que la Ciudad-Región es entendida por lógicas económicas, el proceso por el cual se hace visible es entendido por lógicas políticas.

En el valle de Aburrá, estas lógicas políticas obedecen a reglas de juego informales, que dominan el panorama institucional sobre las reglas de juego formales. El dominio de las reglas informales sobre las formales existe en el caso del manejo de los flujos económicos entre jurisdicciones político-administrativas, debido a las limitaciones de ejecución presupuestal mencionadas por Yory (2011). Es por eso que la interdependencia entre el Área Metropolitana y su entorno regional es un problema que enfrenta el distrito y que encuentra un espacio político marcado sobre todo por la voluntad de los actores a cooperar.

Sin embargo, a diferencia de los casos norteamericanos, chilenos y mexicanos documentados por Hawkins (2010) y por Orellana (2012, 2009) respectivamente, la fractura de los gobiernos locales no es tan fuerte en el valle, toda vez que, desde 1980, ha logrado configurarse el Área Metropolitana del Valle de Aburrá (AMVA) como consecuencia de una asociación de municipios reglamentada hoy por la ley 1625 de 2013.

Esta entidad administrativa del Estado ha venido consolidando su intervención dentro de la jurisdicción político-administrativa de sus asociados en tres grandes temas: la planificación del territorio, la autoridad ambiental y la autoridad de transporte y movilidad.

Pero esta consolidación no ha estado exenta a la política burocrática que definen Muller y Surel (1998). Es decir que, la asignación presupuestal y la intervención en el territorio, dependen de la relación de fuerzas entre los municipios en el marco de la Junta Metropolitana, que es la instancia decisoria de la entidad. Y esta relación de fuerzas, siendo sensible al peso político-electoral y a la disponibilidad de recur- 
sos financieros, es, como lo confirma Leyva (2010), cada vez más dominada por el municipio central, en este caso Medellín.

En cuanto al AMVA, también es cierto que su capacidad para resolver problemas funcionales como por ejemplo el de la movilidad, contrasta con su dificultad para pensar e intervenir de manera holística. De conformidad con lo que observa Wheeler (2009) en el caso de otras áreas metropolitanas en el mundo, el AMVA no se desempeña tan bien cuando se trata de construir agendas regionales.

Ahora bien, si las reglas informales dominan sobre las formales en el proceso de construcción política de la Ciudad-Región, ¿cuál podría ser la lección más relevante de las reglas informales existentes en el territorio para la gobernanza del Distrito? Un buen ejemplo para abordar brevemente esta pregunta es la Comisión Tripartita, pues se trata del acuerdo de voluntades más ambicioso de los últimos diez años respecto al desarrollo del valle de Aburrá y su entorno regional.

La Comisión Tripartita surge en 2004 luego de una declaratoria de trabajo conjunto entre la Gobernación de Antioquia, el Área Metropolitana del Valle de Aburrá y la Alcaldía de Medellín. Fruto de la Comisión se cuenta hoy con dos instrumentos clave para el desarrollo regional: los Lineamientos de Ordenamiento Territorial para Antioquia (LOTA) y el Plan Regional de Competitividad para Medellín, Valle de Aburrá y Antioquia (PRC).

Mientras que el primer instrumento fue realizado en dos fases (2007 y 2012) y aporta elementos para la gestión territorial, el segundo se hizo en 2009 y contribuye sobre todo a la economía urbana. En cuanto a acuerdos, el principal logro de LOTA es la coordinación entre las entidades del Estado en el ámbito local y la negociación de intereses entre éstas y las comunidades locales.

Por su parte, el resultado del PRC que se destaca es la negociación entre la Comisión Tripartita y los gremios empresariales, gracias a la mediación de la Cámara de Comercio de Medellín para Antioquia en el marco de la Comisión Regional de Competitividad de Antioquia.

No obstante lo anterior, el propósito de integrar la economía y el territorio necesitaría combinar la gestión territorial (LOTA) con la economía urbana (PRC), pues la movilidad del trabajo, del capital y de la información se beneficiarían así de las ventajas competitivas del territorio en sus múltiples escalas geográficas, como es el caso de la ciencia, la tecnología y la innovación: esta es precisamente la configuración de la interdependencia entre la ciudad de Medellín, el valle de Aburra y el entorno regional. 
Si se analiza la génesis de dichos instrumentos, esta integración no parece plantearse con claridad debido a dos inconvenientes estructurales. Primero, la participación del Estado y de los diferentes subsistemas de la sociedad se lee incompleta y, segundo, el fenómeno de aglomeración económica no se entiende en forma de red.

Por un lado, la interacción entre el Estado y la sociedad que demanda la gobernanza no estaría incluyendo subsistemas como el de los gremios económicos pues éstos parecen permanecer al margen de la formulación de LOTA. Por el otro lado, el análisis de la aglomeración económica no estaría pensándose en forma de red como suelen configurarse hoy los flujos, ya que el vínculo con su entorno regional parece limitarse a clústeres empresariales concentrados en Medellín.

Por lo tanto, la lección más relevante del acuerdo voluntario de la Comisión Tripartita para la gobernanza del distrito sería la necesidad de integración de instrumentos asegurando la participación del Estado, los gremios económicos y la comunidad local. En efecto, la gestión de la ciencia, la tecnología y la innovación sería conveniente para que se fortalezcan los recursos y capacidades que el territorio necesitaría para integrarse y desarrollarse.

\subsection{Gestión de la ciencia, la tecnología y la innovación}

Bajo la anterior presentación y de acuerdo con la Norma internacional UNE 166002 publicada por la Asociación Española de Normalización y Certificación -AENOR (2006), a continuación se hace un análisis de los elementos mínimos que debe integrar el distrito en ciencia, tecnología e innovación para desarrollar su gestión en el marco de una estrategia dinamizadora de procesos de Investigación, Desarrollo e innovación (I+D+i) en el ámbito regional.

La norma mencionada es un documento elaborado a partir de la experiencia en gestión de la I+D+i de profesionales y organizaciones relevantes en la temática. En este sentido se propone un enfoque que le permite ser aplicable a cualquier tipo de organización, independiente de su tamaño o del sector económico en que realice su actividad.

Entre los beneficios que aporta la implantación de un sistema de gestión de la I+D+i según el esquema de esta norma en su última versión y la gestión conforme a sus requisitos, se destacan los siguientes, según AENOR (2006, pág. 5):

1. Fomentar y sistematizar las actividades de $\mathrm{I}+\mathrm{D}+i$ que desarrolla la organización;

2. Proporcionar directrices para organizar y gestionar eficazmente la I+D $+i$ de acuerdo con las metas establecidas por la organización; 
3. Asegurar que no se pierden actividades susceptibles de generar tecnologías propias y/o patentes, a través de las cuales se pueden obtener beneficios adicionales por la transferencia de tecnología o por desgravaciones fiscales;

4. Potenciar la I+D+i como un factor diferencial de competitividad y considerarla como tal en los esquemas de reputación corporativa;

5. Ayudar a planificar, organizar y controlar las unidades de $\mathrm{I}+\mathrm{D}+i$, lo cual redunda en un ahorro de recursos y en una mejora de la motivación e implicación de los empleados.

Esta norma permite identificar de manera integral cómo el sistema de gestión se articula con la política local, regional y nacional de ciencia, tecnología e innovación para hacerla operacional en el marco de la infraestructura científica y tecnológica del sistema de innovación regional. A continuación se presentarán cada uno de los requerimientos de la norma y la realidad del sistema regional de innovación en Antioquia frente a este requerimiento.

\section{- Situación actual del sistema regional de innovación en Antioquia frente al requisito del Modelo y sistema de gestión para la I+D+i de la norma AENOR (2006)}

En la actualidad, Ruta N (2014b), con el apoyo del modelo 22@, está desarrollando el modelo con el cual debe operar el distrito en ciencia, tecnología e innovación para la ciudad de Medellín.

Ruta N es una Corporación que ha sido creada por la Alcaldía de Medellín y las Empresas UNE y EPM, y opera como un centro de innovación y negocios que apoya y financia el desarrollo de proyectos para el mejoramiento de la competitividad de Medellín y su área metropolitana. Para lograr esta finalidad, Ruta N abre convocatorias a lo largo del año en temas asociados con los programas y proyectos presentados en la tabla 1.

Tabla 1. Público objetivo y temas de convocatorias Ruta N

\begin{tabular}{|l|l|l|l|}
\hline \multicolumn{1}{|c|}{ Ciudadanos } & \multicolumn{1}{c|}{ Investigadores } & \multicolumn{1}{c|}{ Instituciones } & \multicolumn{1}{c|}{ Empresarios } \\
\hline - Cultura de la innova & - Capital semilla & - Plan CTI & - Capital semilla \\
ción & - Cultura de la innova- & - Medellinnovation & - Ingenio 2013 \\
- Especial EmTech & Ción & - Si lo imaginas es & - Medellinnovation \\
- Social Lab & posible & - Si lo imaginas es po- \\
- Medellinnovation & - Medellíinovation & & \\
- Si lo imaginas es posi $~$ & & \\
ble Si lo imaginas es po & & \\
- ViveLab Medellín & sible & & \\
\hline
\end{tabular}

Fuente: tomado de Ruta N (2014c) 
- Situación actual del sistema regional de innovación en Antioquia frente al requisito de Responsabilidad de la dirección de la norma AENOR (2006)

La Alcaldía de Medellín a través Ruta N lidera el desarrollo del distrito en ciencia, tecnología e innovación. En este sentido se han generado y aprobado las políticas que permiten el desarrollo de este importante proyecto para Medellín y el Área Metropolitana.

Igualmente se cuenta con la participación y el apoyo de universidades, centros de desarrollo tecnológico, incubadoras de empresas, centros regionales de productividad e innovación, parques tecnológicos y empresas como actores fundamentales de las capas que deben integrar e interrelacionarse en el distrito para fomentar el desarrollo de una economía y sociedad del conocimiento.

- Situación actual del sistema regional de innovación en Antioquia frente al requisito de Gestión de recursos de la norma Aenor (2006)

Ruta N cuenta con recursos aprobados por la Alcaldía y el Concejo de Medellín que garantizan su sostenibilidad financiera.

Igualmente se cuenta con la infraestructura científica y tecnológica en la región que permite impulsar los clústeres estratégicos (Energía, Salud, TIC) para la ciudad, pero es necesario seguir fortaleciendo tanto los programas de maestría y doctorado (ver Tabla 2) como los grupos de investigación que fomenten el desarrollo para estos clústeres estratégicos (ver figura 1).

Tabla 2.Número de programas de formación de maestría y doctorado de las universidades que conforman el Comité Universidad Empresa Estado en Antioquia

y se destacan en actividades de $\mathrm{I}+\mathrm{D}+\mathrm{i}$

\begin{tabular}{|c|c|c|c|c|c|c|c|c|c|c|c|c|c|c|c|c|}
\hline \multicolumn{17}{|c|}{$\begin{array}{c}\text { Capacidades de las universidades del G8 que fortalecen el aprendizaje científico y tecnológico } \\
\text { para sectores estratégicos de Medellín }\end{array}$} \\
\hline \multirow{2}{*}{$\begin{array}{l}\text { Cadena } \\
\text { industrial }\end{array}$} & \multicolumn{2}{|c|}{ UdeA } & \multicolumn{2}{|c|}{ Unal } & \multicolumn{2}{|c|}{ UPB } & \multicolumn{2}{|c|}{ Eafit } & \multicolumn{2}{|c|}{ CES } & \multicolumn{2}{|c|}{ La Salle } & \multicolumn{2}{|c|}{ UdeM } & \multicolumn{2}{|c|}{ ElA } \\
\hline & M & D & $M$ & $D$ & $\mathrm{M}$ & $D$ & $\mathrm{M}$ & $D$ & $\mathrm{M}$ & $D$ & M & $\mathrm{D}$ & M & D & M & D \\
\hline E n e rg ía & 1 & 1 & 1 & 2 & 1 & 1 & 0 & 0 & 0 & 0 & 0 & 0 & 0 & 0 & 0 & 0 \\
\hline Salud & 34 & 11 & 0 & 0 & 2 & 1 & 0 & 0 & 5 & 1 & 0 & 0 & 0 & 0 & 2 & 0 \\
\hline TIC & 2 & 1 & 1 & 0 & 2 & 1 & 1 & 1 & 0 & 0 & 0 & 0 & 3 & 0 & 0 & 0 \\
\hline
\end{tabular}

Fuente: elaboración propia con información disponible en la web de las universidades. 
Figura 1. Grupos de investigación en áreas estratégicas (Energía, Salud y TIC) de las universidades que conforman el Comité Universidad Empresa Estado (CUEE) en Antioquia
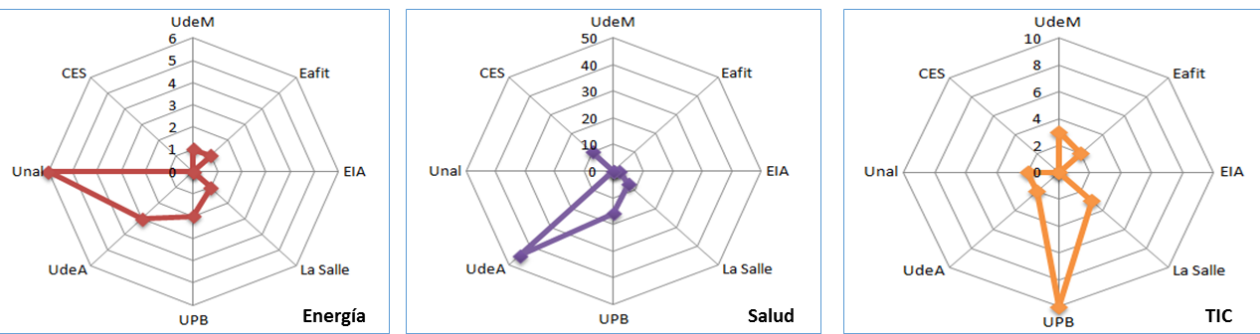

Fuente: elaboración propia a partir de Colciencias (2013)

La tabla 2 refleja la baja oferta en programas de formación de alto nivel (maestrías y doctorados) asociados a los sectores estratégicos para Medellín. Solo se destaca la oferta en programas asociados al área de la salud por parte de la Universidad de Antioquia (2013) y la Universidad CES (2013).

La figura 1 muestra las siguientes debilidades y fortalezas de cara al desarrollo de los clústeres estratégicos de Medellín. Por un lado, solo existen 13 grupos de investigación (11.3\%) en el área de energía pertenecientes a las universidades que conforman el CUEE frente a un total de 115 grupos de investigación de estas mismas instituciones. Esta situación refleja una debilidad frente al desarrollo de un clúster tan importante para la ciudad como es de energía.

Asimismo, en el área estratégica de TIC se muestra que solo existen 22 grupos de investigación (19.1\%), pertenecientes a las universidades que conforman el CUEE frente a un total de 115 grupos de investigación de estas mismas instituciones. Esta situación refleja una debilidad frente al desarrollo de un clúster tan importante para la ciudad como es el de TIC.

Por el otro, en el área estratégica de Salud existen 80 grupos de investigación (69.6\%) pertenecientes a las universidades que conforman el CUEE frente a un total de 115 grupos de investigación de estas mismas instituciones. Esta situación refleja una fortaleza frente al desarrollo de un clúster tan importante para la ciudad como es de salud.

En Antioquia, para un total de 737 grupos de investigación pertenecientes a las Instituciones de Educación Superior, solo se cuentan con 115 grupos de investigación (15\%) en las áreas de energía, salud y TIC, las cuales representan sectores de gran importancia para el desarrollo del plan estratégico de ciencia, tecnología e innovación (2011-2021) para Medellín. 
- Situación actual del sistema regional de innovación en Antioquia frente al requisito de Actividades de I+D+i de la norma AENOR (2006)

En Medellín se cuenta con un Plan Estratégico de ciencia, tecnología e innovación (2011-2021) que identifica las brechas tecnológicas y oportunidades de negocio que pueden ser aprovechadas por la infraestructura científica y tecnológica para potenciar el desarrollo del distrito en ciencia, tecnología e innovación, como lo plantea RUTA N (2014d).

\section{- Situación actual del sistema regional de innovación en Antioquia frente al requisito de Medición, análisis y mejora de la norma AENOR (2006)}

Finalmente, en la actualidad la gran mayoría de las entidades que conformarán el distrito cuentan con sistemas de seguimiento y medición de sus procesos de I+D+i como parte de su sistema de gestión, pero no cuentan con sistemas de seguimiento y medición de los resultados del proceso de I+D+i como una actividad estratégica que permita verificar que los impactos generados efectivamente están orientando a Medellín y su Área Metropolitana hacia una economía y sociedad del conocimiento.

\section{CONCLUSIONES}

El área metropolitana del valle de Aburrá es un territorio en el cual la ciencia, la tecnología y la innovación son factores estratégicos de desarrollo. Por tanto, el nuevo distrito busca aprovechar estos factores y fomentar capacidades y competencias sobre la base del conocimiento.

Este propósito se traduce en la integración de la economía y el territorio y en el relacionamiento de los actores que allí intervienen, es decir, en la gobernanza del distrito. Tres son las bases fundamentales de esta gobernanza: tener claridad sobre las dinámicas y tendencias del territorio, relacionar los flujos económicos con las lógicas políticas y gestionar la ciencia, la tecnología y la innovación.

En primer lugar, las dinámicas y tendencias del territorio están orientadas hacia la construcción de Ciudad-Región, fomentando la educación a través de redes y creando capacidades de transformación. Es decir que esta construcción se hace sobre la base de una cultura de la innovación dentro de las instituciones de educación técnica, tecnológica y superior y dentro de las empresas en general. Por tanto, debe existir unión e intercambio de información entre el sector empresarial, las instituciones educativas y la sociedad civil. 
En segundo lugar, se deben articular las lógicas económica y política inherentes al área metropolitana y su entorno regional por medio del Distrito como arreglo institucional. Mientras que las lógicas económicas obedecen a flujos definidos por la movilidad del trabajo, del capital y de la información, las lógicas políticas son relacionales y se ajustan a fronteras territoriales.

En el caso del valle de Aburrá y su entorno regional, se observa que dicha integración necesita sobre todo los acuerdos voluntarios, dadas las limitaciones normativas. Estos acuerdos deberían procurar, en cualquier actividad, la participación del Estado y de, al menos, dos grandes subsistemas de la sociedad: los gremios económicos y la comunidad local. En efecto, esta participación debería existir tanto en los ejercicios de gestión territorial a escala regional, como en aquellos asociados a la economía urbana.

Es por eso que el Distrito debería velar por el fortalecimiento de la dimensión económica de la gestión territorial y por una economía urbana que considere sus vínculos con los recursos y capacidades de la ciudad y de su entorno regional. Esto es que el Distrito se construya sobre la base de la ciencia, la tecnología y la innovación, procurando la integración de instrumentos de desarrollo regional como los Lineamientos de Ordenamiento Territorial para Antioquia y el Plan Regional de Competitividad para Medellín, Valle de Aburrá y Antioquia.

Finalmente, de acuerdo con lo expresado Josep Miquel Piqué, se vienen dando las condiciones para la construcción del modelo y sistema de gestión del distrito a través de entidades como Ruta $\mathrm{N}$ y de la dinámica de diferentes agentes del sistema regional de ciencia, tecnología e innovación. Sin embargo, es evidente que se tienen debilidades desde la gestión de los recursos en la oferta de programas de formación de alto nivel (maestrías y doctorados) y grupos de investigación en las áreas donde se proyectan los clústeres estratégicos (energía, salud y TIC) para Medellín.

Igualmente se identifica la necesidad de parte de los agentes del sistema regional de ciencia, tecnología e innovación, de crear sistemas de seguimiento y medición de los resultados del proceso de I+D+i como una actividad estratégica que permita verificar que los impactos generados efectivamente están orientando a Medellín y su área metropolitana como una economía y sociedad del conocimiento.

Investigaciones futuras deben orientarse hacia la generación de nuevos modelos y sistemas que permitan no solo la creación, sino la sostenibilidad de infraestructuras científicas y tecnológicas que permitan hacia verdaderas sociedades y economías del conocimiento. 


\section{BIBLIOGRAFÍA}

AENOR- Asociación Española de Normalización y Certificación (2006). Norma UNE 166002. Gestión de la I+D+i: Requisitos del Sistema de Gestión de la I+D+i. Asociación Española de Normalización y Certificación, Madrid, p. 22

Agudelo, S., Medina J., Álvarez-García, L. (2010). La proyección institucional del Valle de Aburrá al 2020. En Semestre económico, 13 (26), 33-56

AMVA - Área Metropolitana del Valle de Aburrá (2007). Plan Integral de Desarrollo Metropolitano. Metrópoli 2008-2020. Hacia la Integración Regional Sostenible. Medellín, AMVA, p. 280

Brundtland, G. H. (1987). Our Common Future. Tokyo, World Commission on Environment and Development, p. 300

Comisión Tripartita (2012). Lineamientos de Ordenamiento Territorial para Antioquia Fase II. Construyendo Juntos Acuerdos Territoriales. Medellín, Gobernación de Antioquia, Área Metropolitana del Valle de Aburrá, Municipio de Medellín, p. 157

Comisión Tripartita (2007). Lineamientos de Ordenamiento Territorial para Antioquia Fase I. Propuesta Pública para Discusión. Medellín, Gobernación de Antioquia, Área Metropolitana del Valle de Aburrá, Municipio de Medellín, p. 382

CRC-Congreso de la República de Colombia (2011). Ley 1454 del 28 de junio de 2011: Por la cual se dictan normas orgánicas sobre ordenamiento territorial y se modifican otras disposiciones. Diario Oficial No. 48.115 del 29 de junio de 2011

CRC-Congreso de la República de Colombia (2013). Ley 1617 del 05 de febrero de 2013: Por la cual se expide el régimen para los Distritos especiales. Diario Oficial No. 48.695 de 5 de febrero de 2013.

CRC-Congreso de la República de Colombia (2013). Ley 1625 del 29 de abril de 2013: Por la cual se deroga la Ley Orgánica 128 de 1994 y se expide el Régimen para las Áreas Metropolitanas. Diario Oficial No. 48.776 de 29 de abril de 2013.

CUEE - Comité Universidad Empresa Estado de Antioquia (2013). Página web institucional [Consultado: junio de 2013]

Consejo de Medellín (2008). Acuerdo municipal No 55.m [Consultado: 24 de abril de 2012]

Colciencias- Departamento Administrativo de Ciencia, Tecnología e Innovación (2013). Plataforma

Scienti - Colombia: Grupos de investigación en Antioquia. [Consultado: 10 de junio de 2013]

DNP- Departamento Nacional de Planeación (2000). Agenda de conectividad. Conpes 3072. [Consultado: Abril 27 de 2012] 
Bases para la gobernanza del distrito de ciencia, tecnología e innovacion en Medellín, Colombia

Departamento Nacional de Planeación [DNP] (2007). Plan Nacional de Desarrollo Científico, Tecnológico y de innovación. Colciencias, 2007-2019. [Consultado: Agosto 1 de 2013]

Departamento Nacional de Planeación [DNP] (2010). Plan nacional de desarrollo 2010-2014. [Consultado: Agosto 1 de 2013]

Dovers, S. R., Hezri, A. A. (2010). Institutions and policy processes: the means to the ends of adaptation. En Wiley Interdisciplinary Review - Climate Change, 1 (2), 212-231. DOI: 10.1002/ wcc. 29

Fischer, A., Petersen, L., Feldkoetter, C. (2007). Sustainable governance of natural resources and institutional change, an analytical framework. En Public Administration and Development, 27 (2), 123-137. DOI: 10.1002/pad.442

Gobernación de Antioquia (2012). Plan de desarrollo del departamento de Antioquia (2012-2015). Medellín, Gobernación de Antioquia. [Consultado: Diciembre 15 de 2015]

Harrison, J. (2010). Networks of connectivity, territorial fragmentation, uneven development: The new politics of city-regionalism. En Political Geography, 29, 17-27. DOI: 10.1016/j. polgeo.2009.12.002

Hawkins, Ch. (2010). Competition and cooperation: local government joint ventures for economic development. En Journal of Urban Affairs, 32 (2), 253-275. DOI: 10.1111/j.1467$-9906.2009 .00492 . x$

Launay, C. (2006). El uso del concepto de gobernanza y/o gobernabilidad en Colombia. IRG. [Consultado: Julio 1 de 2010]

Le Galès, P. (1995). Du gouvernement des villes à la gouvernance urbaine. En Revue Française de Sciences Politiques, 45 (1), 57-95.

Leyva, S. (2010). El proceso de construcción de estatalidad local (1998-2009): ċla clave para entender el cambio de Medellín? In: Hermelin, M., Echeverri, A., Giraldo, J. (Eds.), Medellín, Medio ambiente, Urbanismo, Sociedad, Ed. URBAM-EAFIT, Medellín. pp. 271-293.

Massiris-Cabeza, A. (1993). Racionalidad espacial de la actual división político-administrativa de Colombia: Hacia una nueva regionalización departamental, Trabajo de grado para optar el título de Magister en Geografía, Convenio UPTC-IGAC, Bogotá

Massiris-Cabeza, A. (2011). Ordenamiento territorial y procesos de construcción regional. Biblioteca virtual Luis Ángel Arango, Bogotá. [Consultado: Marzo 7 de 2011]

Ministerio de Educación Nacional [MEN] (2011). Proyecto de reforma a la Educación superior. [Consultado: Diciembre 15 de 2015] 
Molina-Betancur, C.; Polanco, J. A. (2015). Un desafío de sostenibilidad: el nuevo distrito metropolitano de ciencia, tecnología, innovación y emprendimiento del valle del Aburrá en Colombia. En Estudios Geográficos, 76 (279), 579-607

Molina-Betancur, C. (2011). El Área Metropolitana del Valle de Aburrá: Hacia una Regionalización Departamental. En Herreño, D., González, E. (Eds.), Memorias II Congreso Internacional Gobernabilidad de las Áreas Metropolitanas. Ed. Universidad de Santo Tomás, Bucaramanga, pp. 19-48.

Muller, P.; Surel, Y. (1998). L'analyse des politiques publiques. Paris, Montchrestien, Clefs politique

North, D. (1991). Institutions. En The Journal of Economic Perspectives, 5 (1), 97-112. DOI:10.1257/ jep.5.1.97.

OECD- Organization for Economic Co-operation and Development (2001). Strategies for sustainable development. Paris, OECD, p. 73

OECD-Organization for Economic Co-operation and Development (2011). Towards Green Growth: Monitoring Progress. Paris, OECD, p. 144

OECD-Organización para la cooperación y el desarrollo económico y Banco Mundial (2012). Evaluación de políticas nacionales de educación. La educación superior en Colombia 2012. Paris, Édition OCDE, p. 350

Orellana, A. (2012). Periurbanización y sustentabilidad en grandes ciudades. En Revista EURE, Reseñas, 38 (115), 247-251.

Orellana, A. (2009). La gobernabilidad metropolitana de Santiago: la dispar relación de poder de los municipios. En Revista EURE, 35 (104), 101-120.

Patiño-Millán, B. (2004). Historia regional de Antioquia. En Consorcio Estudios Regionales en Antioquia (Eds.), Estudios regionales en Antioquia, Editorial La Carreta, Medellín. p. 23-58.

Polanco, J. A. (2009). Un proyecto de territorio para la protección ambiental: entre discurso global y práctica local. En Rosique, J., Gutiérrez, A. L. (Eds.) Estudios sobre territorio, ciudadanía y planeación, Ed. Universidad de Antioquia, Medellín. pp. 63-92.

Prats, J. (2005). De la burocracia al management, del management a la gobernanza. Las transformaciones de las administraciones públicas de nuestro tiempo. Madrid, Instituto Nacional de Administración Pública, p. 209

Rodríguez, O. (2001). Los fundamentos del estructuralismo latinoamericano. En Revista Comercio Exterior, Febrero, 100-112.

Ruta N (2014a). Sobre el modelo de 22@. [Consultado: septiembre 3 de 2014] 
Bases para la gobernanza del distrito de ciencia, tecnología e innovacion en Medellín, Colombia

Ruta N (2014b). Distrito educativo en Ciencia, Tecnología, Innovación y Emprendimiento para Medellín. [Consultado: 10 de enero de 2014]

Ruta N (2014c). Programas y Proyectos para Medellín. [Consultado: 10 de enero de 2014]

RUTA N (2014d). Plan de CTi. [Consultado: 3 de septiembre 2014]

Salmi, J. (2013). La Urgencia de Ir Adelante: Perspectivas desde la Experiencia Internacional para la Transformación de la Educación Superior en Colombia. Bogotá, Ministerio de Educación de Colombia, p. 96

Scott, A. (2001). Globalization and the rise of city-regions. En European Planning Studies, 9 (7), 813-826. DOI: 10.1080/09654310120079788

Soderbaum, P. (2009). Making actors, paradigms and ideologies visible in governance for sustainability. En Sustainable Development, 17 (2), 70-81. DOI: 10.1002/sd.404

Stagl, S. (2007). Theoretical foundations of learning processes for sustainable development. En International Journal of Sustainable Development and World Ecology, 14 (1), 52-62. DOI: $10.1080 / 13504500709469707$

Suelt-Cock, V., Osorio, A. X. (2011). La autonomía local en diversos sistemas de organización, un marco conceptual para el caso colombiano. En Universitas, 122, 117-168

Wall Street Journal (2013). City of the year. [Consultado: 10 de junio de 2013]

Universia (2011). La importancia del inglés en el mundo laboral. [Consultado: 17 de abril de 2012]

Universidad de Antioquia (2013). Programas de posgrado (Maestrías y Doctorados). [10 de junio de 2013|

Universidad CES (2013). Programas de posgrado (Maestrías y Doctorados). [Consultado: 10 de junio de 2013!

Wheeler, S. (2009). Regions, Megaregions and Sustainability. Regional Studies. 43 (6), 863-876. DOI: $10.1080 / 00343400701861344$

Williamson, O. (1996). The Mechanisms of Governance. New York, Oxford University Press

Williamson, O. (2000). The New Institutional Economics: Taking Stock, Looking Ahead. En Journal of Economic Literature, XXXVIII, 595-613. DOI: 10.1257/jel.38.3.595

Yory García, C. M. (2011). Sustentabilidad, Globalización y Desarrollo Territorial Integrado. In: Herreño, D., González, E. (Eds.), Memorias II Congreso Internacional Gobernabilidad de las Áreas Metropolitanas. Ed. Universidad de Santo Tomás, Bucaramanga. p. 49-66. 